\title{
Molecular aggregations and anisotropy photoinduction in organic compounds
}

\author{
Tariel Ebralidze ${ }^{1, ~ *, ~ N a d i a ~ E b r a l i d z e ~}{ }^{1}$, Giorgi Mumladze ${ }^{2}$ \\ ${ }^{1}$ Department of Quantum Optics and Electronics, Vladimer Chavchanidze Institute of Cybernetics of the Georgian Technical University, \\ Tbilisi, Georgia \\ ${ }^{2}$ Department of Computer Engineering Elements and Nanomaterials, Vladimer Chavchanidze Institute of Cybernetics of the Georgian \\ Technical University, Tbilisi, Georgia
}

Email address:

tarebra@yahoo.com (T. Ebralidze),g.mumladze@gtu.ge (G. Mumladze)

\section{To cite this article:}

Tariel Ebralidze, Nadia Ebralidze, Giorgi Mumladze. Molecular Aggregations and Anisotropy Photoinduction in Organic Compounds. Optics. Vol. 3, No. 4, 2014, pp. 33-36. doi: 10.11648/j.optics.20140304.12

\begin{abstract}
The work is dedicated to a study of anisotropy photoinduction during the light-stimulated molecular aggregations in organic compounds. The case of gelatin or polyvinyl films saturated by azo dyes is considered. By using the video and spectral techniques, the time-formation picture of optical image is studied. It is shown that in this case the image is formed in a similar way as in silver emulsions in the process of photographing — "with the grain distribution".
\end{abstract}

Keywords: Anisotropy Photoinduction, Molecular Aggregations

\section{Introduction}

Gelatin or other gel films painted by azo dyes are objects of a deep interest due to their possible application in information optical recording, nonlinear optics, holography etc, which is based on phenomenon of anisotropy optical photo-induction. According to scientists, anisotropy photo-induction in azo dyes is a result of trans- cis-trans photo isomerization process $[1,2]$. It is considered that, during the anisotropy photo-induction in organic compounds, the information is recorded based on the spatial distribution of photo-induced birefringence value. However, according to our understanding along with this, an image formation in film during the anisotropy photoinduction may occur in a different way too, in particular, - by grain distribution, as it occurs in silver emulsion in the process of photographing. However, unlike the process of photographing the particles - molecule aggregates are the anisotropic particles.

For obtaining of more efficiency during the photoinduction of anisotropy, it is necessary that the dye concentration in solution is as high as possible. Nevertheless, the high value of dye concentration in solution surely leads us to self-induction of dye molecules aggregations in solid films, or to create such conditions when the aggregates are induced by the light-stimulation [3-9].

In the works $[7,9]$ there is given that in gelatin film, dyed by the Chrisophenine and Acridine Yellow water solution mixed with 1:1 proportion and dried under room condition, the self-induced ensemble of molecule anisotropic aggregations was obtained. After its irradiating by the non-polarized active light the photodecomposition of aggregates was realized. Then after irradiating the film in same place by linear polarized active light, we obtained the photoinduction of aggregate ensemble with optical axes with same orientation [7], and the optical image formation took place as well. By using of video microscopy, we showed that the image formation process observed here is an analogue of the formation of photo image in the silver emulsions.

In the work [4], the photoinduction of dye molecules anisotropy aggregations during the action of linear polarized light in the gelatin film saturated by azo dye Chrisophenine has been observed.

Here, based on video microscopy filming and spectral measurements the image formation picture during the photoinduction of aggregates in azo dyes is presented.

\section{Experiment}

The experimental measurements were carried out for the polyvinyl film saturated by azo dye "Mordant Pure Yellow" in the dynamic regime of photoinduction anisotropy. The thickness of the prepared film was approximately $200 \mu \mathrm{m}$. Fig. 
1 shows a structural formula of the given dye. The absorption spectrum of the given film is presented below (Fig.2).

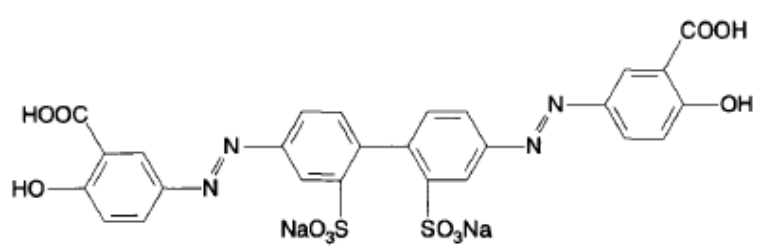

Fig 1. Formula of the dye "Mordant Pure Yellow" (MPY)

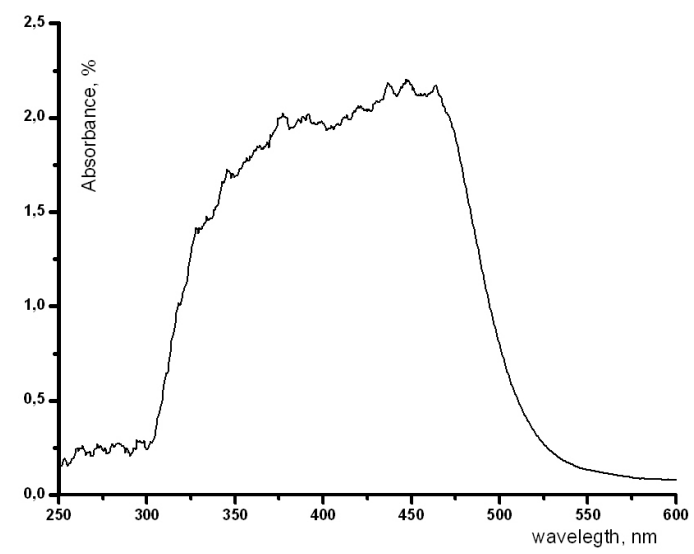

Fig 2. The absorption spectrum of the experimental film

The anisotropy photoinduction in the film was conducted by linearly polarized light of the $100 \mathrm{w} / 2 \mathrm{Hg}$-lamp from a narrow spectral band containing $440 \mathrm{~nm}$ wavelengths. Fig. 3 shows the spectral transmissions of the film after every 30 -second's exposition observed in the crossed polarizers.

As we can see, according to the curves in Fig. 3, there is no anisotropy observed at the beginning, - and the light transmission in the film is not observed,

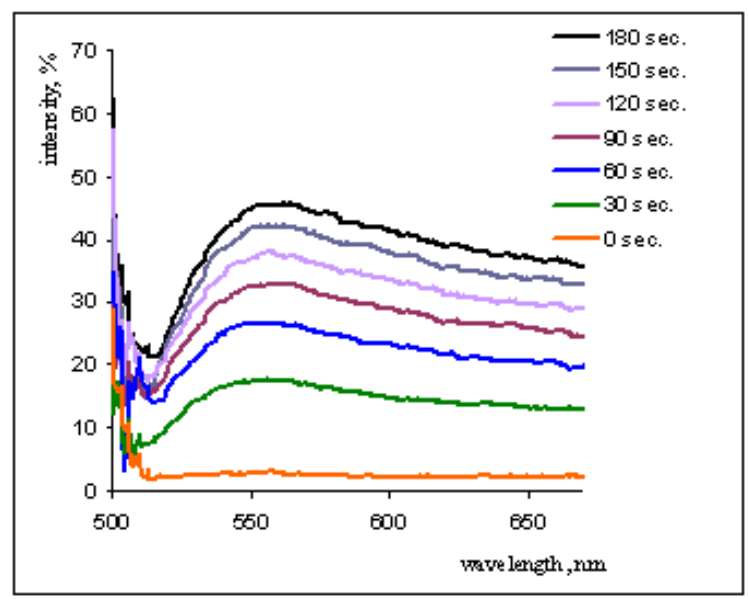

Fig 3. Spectral transmissions of the film with the photoinduced anisotropy in the crossed polarizers duringdifferent expositions

While later, as the expositions increases, the value of anisotropy also increases; however, in the crossed polarizers, the location of the maximum of the curves reflecting the spectral transmission of the film on the spectral axis and shape of the curves do not change.
Below it is shown that the modulation of spectral transmission according to the light exposition shown in Fig. 3 is similar to the modulation of spectral transmission in the crossed polarizers for a crystal phase plate, which is obtained during the modulation of the light-conducting area in the area of measurement.

In order to demonstrate this, below we give the kind of curves reflecting the spectral transmission of a phase plate placed in the crossed polarizers for different portions of anisotropic area of a unit area of measurement. An example of a quartz plate is considered, whose birefringence value is $\Delta n=0,009$ and thickness is $\mathrm{d}=100 \mathrm{mcm}$.

As it is known [10], the spectral transmission of a phase plate placed in the crossed polarizers for the area of measurement with a unit area is given by the formula:

$$
J=\frac{I}{I_{0}}=\sin ^{2} \frac{\pi}{\lambda} \Delta n d
$$

where I is the intensity of light passed in the system (polarizer, plate, analyzer), $I_{0}$ is the initial intensity, $\Delta n$ is the value of birefringence, $\mathrm{d}$ is the plate thickness and $\lambda$ is the light wavelength. It is assumed that the optical axis of the plate creates a $45^{\circ}$ angle with the axis of polarizer.

Expression (1) is written for the area of measurement with an unit area. If not the total area of the unit area of measurement is the light conductor, but only part $\mathrm{C}_{\mathrm{i}}$ thereof, in such a case expression (1) will acquire the following form:

$$
J=C_{i} \sin ^{2} \frac{\pi}{\lambda} \Delta n d
$$

Let us admit that during the experiment, the portion of the anisotropic area in the area of measurement changes and let us investigate how this change is reflected on the curves of spectral transmissions. Let us perform the investigations for the quartz plate with the above-given parameters. If inserting the values of parameters in (2), we will obtain [10]:

$$
J=C_{i} \sin ^{2} \frac{2826}{\lambda}
$$

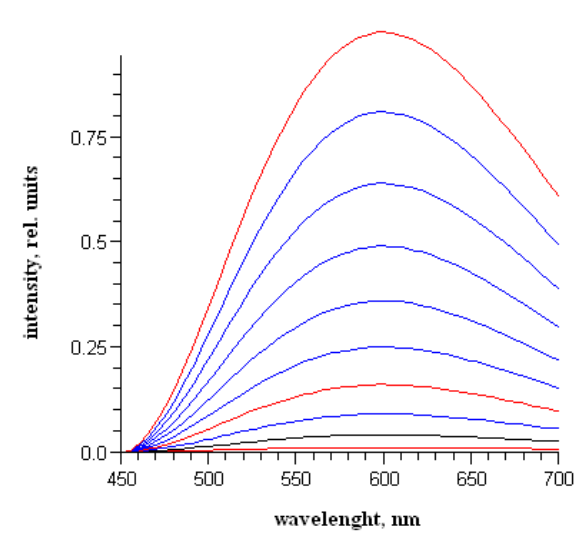

Fig 4. Spectral transmissions of a quartz plate in the crossed polarizers for anisotropic areas of different values in the area of measurement 
Fig. 4 shows the spectral transmissions determined by (3) for different values of $\mathrm{C}_{\mathrm{i}}$.

If comparing the change obtained from the dependence on the exposition of the curves reflecting the spectral transmissions presented in Fig. 3 and change of the curves reflecting the spectral transmissions obtained by modulation of parameter $C_{i}$ for a quartz plate in Fig. 4, we will see that there is a qualitative similarity between them.

Therefore, we conclude that the experimental results shown in Fig. 3 evidence that the portion of the anisotropic area in the area of measurement changes according the exposition of active light in the film, i.e. in this case the image in the film is formed by the distribution of grains, i.e. in the way similar to the image formation in silver emulsions [3].

Hence, we can conclude that modulation of the film transparency during this experiment can take place not only based on the birefringence value modulation, as considered, but also by modulation of the completely integral area of the anisotropic part.

This is directly proved by the digital photos of the film placed between the crossed polarizers, which were made in a dynamic regime of photoinduction of anisotropy.

These digital photos selected out of the series of photos have been shot at the beginning, in the middle and at the end of the exposition, respectively. In case of exposition with linearly polarized active light in the film, at the beginning the anisotropy is not induced over the whole exposed area, but first it is induced in individual areas with their number and areas increasing according to the exposition finally filling the area exposed by light.

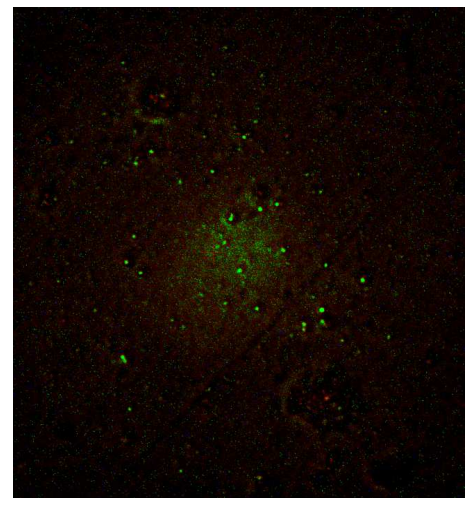

(a)

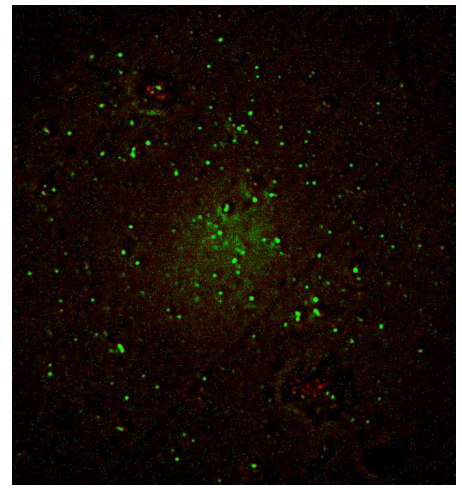

(b)

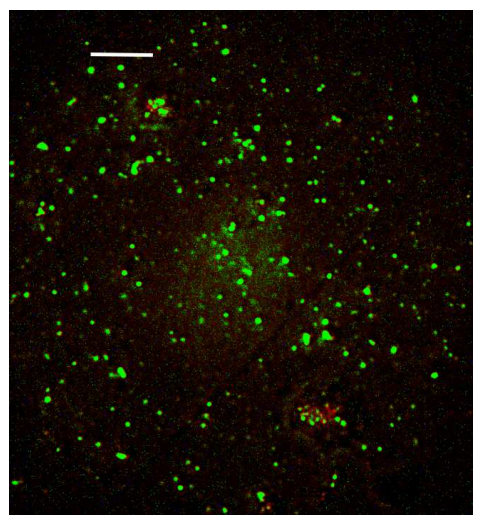

(c)

Fig 5. The image of the area with photoinduced anisotropy observed in the crossed polarizers in the beginning (a), middle (b) and end ( $(c)$, with line length of $20 \mu \mathrm{m}$ ) of exposure.

As we can see in Fig. 5(c), anisotropy photoinduction in the given film took place in individual anisotropic micro-areas, i.e. in "grains" and not over the whole area exposed by light (See the light areas in the figure). Figures 5(a), 5(b) and 5(c) show that the micro-areas, i.e. "grains", in which the anisotropy photoinduction took place, propagate and widen as the exposure time increases what in the final account causes the modulation of their integrated area depending on exposure, i.e. in the given case, the optical image in the film is recorded in the way similar to that of recording the image in silver emulsions, with the distribution of the total area of the "grains". We are given a certain imagination of the sizes of the "grains" in Fig. 5(c) by the image of a $20 \mu \mathrm{m}$ long line.

\section{Conclusion}

Thus, for Polyvinyl Alcohol saturated by azo dye Mordant Pure Yellow has been shown, that the anisotropy photoinduction can occur therein by the photoinduction of molecular aggregations so, that the optical image in the film would be recorded as distribution of grains, similar photography of silver emulsions.

\section{Acknowledgment}

Authors express gratitude for participation in the theoretical analysis to Professor Grigor Giorgadze.

\section{References}

[1] A. M. Makushenko, B. S. Neporent, B. S. Neporent, and O. V. Stolbova, "Reverssible orientational photodichroism and photoisomerization of aromatic azo compounds. I," Optics and Spectroscopy (USSR), vol. 31, pp. 557-564, 1971.

[2] F. L. Labarthet, S. Freiberg, C. Pellerin, M. Pézolet, A. Natansohn, and P. Rochon, "Spectroscopic and optical characterization of a series of azobenzene-containing side-chain liquid crystalline polymers," Macromolecules, vol. 33 , no. 18 , pp. 6815-6823, 2000. 
[3] T. D. Ebralidze and A. N. Mumladze, "Light-induced anisotropy in azo-dye-colored materials," Applied Optics, vol. 29, no. 4, pp. 446-447, 1990.

[4] T. D. Ebralidze, N. A. Ebralidze, A. N. Mumladze, "Photoinduction of anisotropic grains in organic compounds", Applied Optics, 37, 6161-6163, 1998.

[5] D. Voloschenko and O. D. Lavrentovich, "Light-induced director-controlled microassembly of dye molecules from a liquid crystal matrix," Journal of Applied Physics, vol. 86, no. 9, pp. 4843-4846, 1999.

[6] B. J. Kim, S. Y. Park, and D. H. Choi, "Effect of molecular aggregation on the photo-induced anisotropy in amorphous polymethacrylate bearing an aminonitroazobenzene moiety," Bulletin of the Korean Chemical Society, vol. 22, no. 3, pp. 271-275, 2001.
[7] T. D. Ebralidze, N. A. Ebralidze, G. A. Mumladze, and E. S. Kitsmarishvili, "Light-controlled mass formation of aggregates of molecules in organic compounds," Chinese Optics Letters, vol. 7, no. 9, pp. 823-825, 2009.

[8] A. Priimagi, A. Shevchenko, M. Kaivola, F. J. Rodriguez, M. Kauranen, and P. Rochon, "High and stable photoinduced anisotropy in guest-host polymer mediated by chromophore aggregation," Optics Letters, vol. 35, no. 11, pp. 1813-1815, 2010 .

[9] T. D. Ebralidze, N. A. Ebralidze, and G. A. Mumladze, "Anisotropy Photoinduction during the Mass Associations of Dye Molecules in Gelatin Films", Journal of Materials, Volume 2013, Article ID 507938, 3 pages, 2013.

[10] A.V. Shubnikov, "Bases of the Optical Crystallography", Publishing House of the Academy of Sciences, USSR, 1958. 